Supporting Information for

\title{
Polarizable Empirical Force Field for Alkanes Based on the Classical Drude Oscillator Model
}

Igor V. Vorobyov, Victor M. Anisimov and Alexander D. MacKerell, Jr.*

Department of Pharmaceutical Sciences, School of Pharmacy, University of Maryland, Baltimore, MD, USA.

*Corresponding author phone: (410)706-7442; fax: (410)706-5017; e-mail: amackere@rx.umaryland.edu. Corresponding author address: 20 Penn Street, Baltimore, MD 21201. 
Table S1. Selected geometric parameters of alkanes

\begin{tabular}{|c|c|c|c|c|c|}
\hline & Exper & $\mathrm{C} 27 \mathrm{r}$ & Diff & Drude & Diff \\
\hline \multicolumn{6}{|l|}{ Ethane } \\
\hline $\mathrm{C}-\mathrm{H}$ & 1.112 & 1.112 & 0.000 & 1.111 & -0.001 \\
\hline $\mathrm{C}-\mathrm{C}$ & 1.534 & 1.533 & -0.001 & 1.531 & -0.003 \\
\hline $\mathrm{H}-\mathrm{C}-\mathrm{H}$ & 107.5 & 108.3 & 0.8 & 108.5 & 1.0 \\
\hline $\mathrm{H}-\mathrm{C}-\mathrm{C}$ & 111.2 & 110.6 & -0.6 & 110.5 & -0.7 \\
\hline \multicolumn{6}{|l|}{ Propane } \\
\hline $\mathrm{C} 1-\mathrm{H}$ & 1.107 & 1.111 & 0.004 & 1.111 & 0.004 \\
\hline $\mathrm{C} 2-\mathrm{H}$ & 1.115 & 1.115 & 0.000 & 1.114 & -0.001 \\
\hline $\mathrm{C}-\mathrm{C}$ & 1.533 & 1.530 & -0.003 & 1.531 & -0.002 \\
\hline $\mathrm{H}-\mathrm{C} 1-\mathrm{H}$ & & 108.3 & & 108.4 & \\
\hline $\mathrm{H}-\mathrm{C} 2-\mathrm{H}$ & 107.8 & 107.4 & -0.4 & 107.5 & -0.3 \\
\hline C-C-C & 112.0 & 111.9 & -0.1 & 112.5 & 0.5 \\
\hline C-C2-H & & 109.4 & & 109.2 & \\
\hline $\mathrm{C}-\mathrm{C} 1-\mathrm{H}$ & & 110.5 & & 110.4 & \\
\hline \multicolumn{6}{|l|}{ Butane } \\
\hline $\mathrm{C} 1-\mathrm{H}$ & 1.117 & 1.111 & -0.006 & 1.111 & -0.006 \\
\hline $\mathrm{C} 2-\mathrm{H}$ & 1.117 & 1.114 & -0.003 & 1.114 & -0.003 \\
\hline $\mathrm{C} 1-\mathrm{C} 2$ & 1.531 & 1.531 & 0.000 & 1.531 & 0.000 \\
\hline $\mathrm{C} 2-\mathrm{C} 3$ & 1.531 & 1.533 & 0.002 & 1.534 & 0.003 \\
\hline $\mathrm{C} 2-\mathrm{C} 1-\mathrm{H}$ & & 110.5 & & 110.4 & \\
\hline $\mathrm{C} 1-\mathrm{C} 2-\mathrm{H}$ & & 109.3 & & 109.1 & \\
\hline H-C2-H & & 107.3 & & 107.4 & \\
\hline C-C-C & 113.3 & 113.3 & 0.0 & 113.6 & 0.3 \\
\hline \multicolumn{6}{|l|}{ Isobutane } \\
\hline Me-H & 1.092 & 1.111 & 0.019 & 1.111 & 0.019 \\
\hline $\mathrm{Me}-\mathrm{Ha}$ & 1.100 & 1.111 & 0.011 & 1.111 & 0.011 \\
\hline $\mathrm{C}-\mathrm{H}$ & 1.108 & 1.118 & 0.010 & 1.117 & 0.009 \\
\hline $\mathrm{C}-\mathrm{C}$ & 1.525 & 1.534 & 0.009 & 1.538 & 0.013 \\
\hline C-Me-H & 109.5 & 110.4 & 1.0 & 110.4 & 1.0 \\
\hline C-Me-Ha & 109.5 & 110.3 & 0.8 & 110.2 & 0.7 \\
\hline H-Me-Ha & 107.9 & 108.5 & 0.6 & 108.6 & 0.7 \\
\hline H-Me-H & 108.5 & 108.6 & 0.1 & 108.6 & 0.1 \\
\hline Me-C-H & 109.4 & 108.2 & -1.2 & 107.9 & -1.5 \\
\hline $\mathrm{C}-\mathrm{C}-\mathrm{C}$ & 111.2 & 110.7 & -0.4 & 111.0 & -0.1 \\
\hline
\end{tabular}

Bond lengths are in $\AA$, bond angles are in degress. Experimental data are from Refs. 1-5. 
Table S2. Comparison of calculated and experimental frequencies for ethane

\begin{tabular}{lllllll}
\hline$\#$ & Assignment & exper & C27r & \%diff & Drude & \%diff \\
\hline 1 & CC tor & 279 & 306 & 9.6 & 305 & 9.3 \\
2 & CH3 rock & 822 & 923 & 12.2 & 919 & 11.8 \\
3 & CH3 rock & 822 & 923 & 12.2 & 919 & 11.8 \\
4 & CC str & 995 & 1002 & 0.7 & 1001 & 0.6 \\
5 & CH3 rock & 1190 & 1113 & -6.5 & 1113 & -6.5 \\
6 & CH3 rock & 1190 & 1113 & -6.5 & 1113 & -6.5 \\
7 & CH3 adef & 1370 & 1416 & 3.4 & 1417 & 3.4 \\
8 & CH3 adef & 1388 & 1416 & 2.0 & 1417 & 2.1 \\
9 & CH3 adef & 1469 & 1436 & -2.2 & 1432 & -2.5 \\
10 & CH3 adef & 1469 & 1436 & -2.2 & 1434 & -2.4 \\
11 & CH3 sdef & 1460 & 1438 & -1.5 & 1438 & -1.5 \\
12 & CH3 sdef & 1460 & 1440 & -1.4 & 1438 & -1.5 \\
13 & CH3 sstr & 2915 & 2895 & -0.7 & 2894 & -0.7 \\
14 & CH3 sstr & 2915 & 2909 & -0.2 & 2909 & -0.2 \\
15 & CH3 astr & 2950 & 2953 & 0.1 & 2953 & 0.1 \\
16 & CH3 astr & 2950 & 2953 & 0.1 & 2953 & 0.1 \\
17 & CH3 astr & 2974 & 2964 & -0.3 & 2964 & -0.3 \\
18 & CH3 astr & 2974 & 2964 & -0.3 & 2964 & -0.3 \\
\hline Freq
\end{tabular}

Frequencies are in $\mathrm{cm}^{-1} . \%$ diff $=((\text { calc.-exp. }) / \text { exp. })^{*} 100$. Experimental data are from Refs. 5-8. 
Table S3. Comparison of calculated and experimental frequencies for propane

\begin{tabular}{lllllll}
\hline$\#$ & Assignment & exper & C27r & \%diff & Drude & \%diff \\
\hline 1 & CCCH tor & 217 & 224 & 3.4 & 225 & 3.7 \\
2 & CCCH tor & 265 & 258 & -2.7 & 257 & -3.0 \\
3 & CCCscis & 375 & 376 & 0.3 & 377 & 0.5 \\
4 & CH2 rock & 748 & 801 & 7.1 & 796 & 6.4 \\
5 & CC str & 868 & 871 & 0.3 & 869 & 0.2 \\
6 & CH3 rock & 921 & 949 & 3.0 & 950 & 3.1 \\
7 & CH3 rock & 899 & 961 & 6.9 & 962 & 7.0 \\
8 & CC str & 1049 & 1061 & 1.2 & 1063 & 1.3 \\
9 & CH3 rock & 1157 & 1089 & -5.9 & 1087 & -6.0 \\
10 & CC str & 1187 & 1097 & -7.6 & 1095 & -7.8 \\
11 & CH2 twis & 1278 & 1214 & -5.0 & 1217 & -4.8 \\
12 & CH2 wag & 1332 & 1381 & 3.6 & 1382 & 3.8 \\
13 & CH3 sdef & 1370 & 1413 & 3.1 & 1410 & 2.9 \\
14 & CH3 sdef & 1385 & 1413 & 2.0 & 1410 & 1.8 \\
15 & CH3 adef & & 1421 & & 1420 & \\
16 & CH3 adef & 1449 & 1424 & -1.7 & 1425 & -1.6 \\
17 & CH3 adef & 1459 & 1425 & -2.4 & 1426 & -2.3 \\
18 & CH3 adef & 1464 & 1428 & -2.5 & 1428 & -2.5 \\
19 & CH2 scis & 1473 & 1447 & -1.7 & 1446 & -1.8 \\
20 & CH2 sstr & & 2895 & & 2894 & \\
21 & CH3 sstr & 2875 & 2901 & 0.9 & 2901 & 0.9 \\
22 & CH2 sstr & 2875 & 2909 & 1.2 & 2909 & 1.2 \\
23 & CH2 astr & 2915 & 2929 & 0.5 & 2929 & 0.5 \\
24 & CH3 astr & 2965 & 2958 & -0.3 & 2958 & -0.2 \\
25 & CH3 astr & 2965 & 2958 & -0.2 & 2958 & -0.2 \\
26 & CH3 astr & & 2960 & & 2960 & \\
27 & CH3 astr & 2965 & 2961 & -0.1 & 2961 & -0.1 \\
\hline & Ceq & & \\
1 & &
\end{tabular}

Frequencies are in $\mathrm{cm}^{-1} . \%$ diff $=(($ calc.-exp. $) /$ exp. $) * 100$. Experimental data are from Refs. 5-8. 
Table S4. Comparison of calculated and experimental frequencies for butane

\begin{tabular}{lllllll}
\hline$\#$ & Assignment & exper & C27r & \%diff & Drude & \%diff \\
\hline 1 & CCCC tor & 121 & 126 & 3.8 & 135 & 11.7 \\
2 & CCCH tor & 0 & 222 & 0.0 & 222 & 0.0 \\
3 & CCCH tor & 266 & 258 & -3.2 & 258 & -3.1 \\
4 & CCCscis & 0 & 292 & 0.0 & 293 & 0.0 \\
5 & CCCscis & 427 & 394 & -7.6 & 395 & -7.4 \\
6 & CH2 rock & 733 & 746 & 1.8 & 745 & 1.6 \\
7 & CH2 rock & 0 & 831 & 0.0 & 829 & 0.0 \\
8 & C2C3str & 835 & 868 & 3.9 & 867 & 3.9 \\
9 & CH3 rock & 944 & 987 & 4.6 & 987 & 4.6 \\
10 & CH3 rock & 965 & 992 & 2.8 & 993 & 2.9 \\
11 & C1C2str & 1010 & 1014 & 0.4 & 1013 & 0.3 \\
12 & C1C2str & 1053 & 1039 & -1.3 & 1040 & -1.2 \\
13 & CH3 rock & & 1058 & & 1058 & \\
14 & C2C3str & 1148 & 1110 & -3.4 & 1109 & -3.4 \\
15 & CH2 twis & 1257 & 1164 & -7.4 & 1166 & -7.3 \\
16 & CH2 twis & 1300 & 1204 & -7.4 & 1205 & -7.3 \\
17 & CH2 wag & 1293 & 1294 & 0.1 & 1295 & 0.1 \\
18 & CH2 wag & & 1353 & & 1354 & \\
19 & CH3 sdef & 1455 & 1411 & -3.0 & 1409 & -3.2 \\
20 & CH3 sdef & 1455 & 1413 & -2.9 & 1410 & -3.1 \\
21 & CH3 adef & & 1419 & & 1420 & \\
22 & CH3 adef & 1460 & 1425 & -2.4 & 1425 & -2.4 \\
23 & CH3 adef & 1462 & 1426 & -2.5 & 1426 & -2.4 \\
24 & CH3 adef & 1468 & 1426 & -2.8 & 1427 & -2.8 \\
25 & CH2 scis & 1375 & 1439 & 4.7 & 1438 & 4.6 \\
26 & CH2 scis & 1459 & 1444 & -1.0 & 1443 & -1.1 \\
27 & CH2 sstr & 2872 & 2893 & 0.7 & 2893 & 0.7 \\
28 & CH3 sstr & 2853 & 2898 & 1.6 & 2898 & 1.6 \\
29 & CH3 sstr & 2861 & 2904 & 1.5 & 2904 & 1.5 \\
30 & CH2 sstr & 2875 & 2910 & 1.2 & 2909 & 1.2 \\
31 & CH2 astr & 2912 & 2924 & 0.4 & 2924 & 0.4 \\
32 & CH2 astr & 2920 & 2934 & 0.5 & 2935 & 0.5 \\
33 & CH3 astr & 2965 & 2959 & -0.2 & 2959 & -0.2 \\
34 & CH3 astr & 2965 & 2959 & -0.2 & 2959 & -0.2 \\
35 & CH3 astr & 2966 & 2959 & -0.2 & 2959 & -0.2 \\
36 & CH3 astr & 2966 & 2960 & -0.2 & 2960 & -0.2 \\
\hline
\end{tabular}

Frequencies are in $\mathrm{cm}^{-1} . \%$ diff $=((\text { calc.-exp. }) / \text { exp. })^{*} 100$.

Experimental data are from Refs. 5-8. 
Table S5. Comparison of calculated and experimental frequencies for isobutane

\begin{tabular}{|c|c|c|c|c|c|c|}
\hline$\#$ & Assignment & exper & $\mathrm{C} 27 \mathrm{r}$ & \%diff & Drude & \%diff \\
\hline 1 & $\mathrm{HCCH}$ tor & 198 & 247 & 24.7 & 249 & 25.8 \\
\hline 2 & $\mathrm{HCCH}$ tor & 0 & 263 & 0.0 & 262 & 0.0 \\
\hline 3 & $\mathrm{HCCH}$ tor & 0 & 263 & 0.0 & 262 & 0.0 \\
\hline 4 & CCCdef & 367 & 362 & -1.5 & 362 & -1.3 \\
\hline 5 & CCCdef & 367 & 362 & -1.5 & 362 & -1.3 \\
\hline 6 & CCCdef & 426 & 428 & 0.4 & 426 & 0.1 \\
\hline 7 & CC str & 797 & 794 & -0.4 & 793 & -0.5 \\
\hline 8 & $\mathrm{CH} 3$ rock & 918 & 916 & -0.2 & 917 & -0.1 \\
\hline 9 & CC str & 918 & 916 & -0.2 & 917 & -0.1 \\
\hline 10 & $\mathrm{CH} 3$ rock & 966 & 958 & -0.8 & 960 & -0.7 \\
\hline 11 & $\mathrm{CH} 3$ rock & 966 & 958 & -0.8 & 960 & -0.7 \\
\hline 12 & $\mathrm{CH} 3$ rock & 966 & 960 & -0.7 & 962 & -0.4 \\
\hline 13 & $\mathrm{CH} 3$ rock & 1166 & 1117 & -4.2 & 1114 & -4.5 \\
\hline 14 & CC str & 1166 & 1135 & -2.7 & 1139 & -2.3 \\
\hline 15 & CC str & 1177 & 1135 & -3.6 & 1139 & -3.2 \\
\hline 16 & CHrock & 1330 & 1355 & 1.9 & 1357 & 2.0 \\
\hline 17 & CHrock & 1330 & 1355 & 1.9 & 1357 & 2.0 \\
\hline 18 & CH3 sdef & 1371 & 1398 & 2.0 & 1397 & 1.9 \\
\hline 19 & CH3 sdef & 1371 & 1398 & 2.0 & 1398 & 2.0 \\
\hline 20 & $\mathrm{CH} 3$ sdef & 1394 & 1399 & 0.4 & 1398 & 0.3 \\
\hline 21 & $\mathrm{CH} 3$ adef & 1475 & 1426 & -3.3 & 1427 & -3.2 \\
\hline 22 & $\mathrm{CH} 3$ adef & 1475 & 1429 & -3.1 & 1430 & -3.1 \\
\hline 23 & $\mathrm{CH} 3$ adef & 1475 & 1429 & -3.1 & 1430 & -3.1 \\
\hline 24 & $\mathrm{CH} 3$ adef & 1477 & 1435 & -2.9 & 1435 & -2.9 \\
\hline 25 & $\mathrm{CH} 3$ adef & 1477 & 1435 & -2.9 & 1435 & -2.9 \\
\hline 26 & $\mathrm{CH} 3$ adef & 1477 & 1436 & -2.8 & 1435 & -2.8 \\
\hline 27 & $\mathrm{CH} 3 \mathrm{str}$ & 2880 & 2900 & 0.7 & 2900 & 0.7 \\
\hline 28 & $\mathrm{CH} 3 \mathrm{str}$ & 2894 & 2900 & 0.2 & 2901 & 0.2 \\
\hline 29 & $\mathrm{CH} 3 \mathrm{str}$ & 2894 & 2901 & 0.2 & 2901 & 0.2 \\
\hline 30 & CHstr & 2904 & 2944 & 1.4 & 2943 & 1.3 \\
\hline 31 & $\mathrm{CH} 3 \mathrm{str}$ & 2962 & 2957 & -0.2 & 2957 & -0.2 \\
\hline 32 & $\mathrm{CH} 3 \mathrm{str}$ & 2962 & 2958 & -0.1 & 2958 & -0.1 \\
\hline 33 & $\mathrm{CH} 3 \mathrm{str}$ & 2962 & 2958 & -0.1 & 2958 & -0.1 \\
\hline 34 & $\mathrm{CH} 3 \mathrm{str}$ & 2962 & 2960 & -0.1 & 2960 & -0.1 \\
\hline 35 & $\mathrm{CH} 3 \mathrm{str}$ & 2962 & 2960 & -0.1 & 2960 & -0.1 \\
\hline 36 & $\mathrm{CH} 3 \mathrm{str}$ & 2962 & 2963 & 0.0 & 2963 & 0.0 \\
\hline
\end{tabular}

Frequencies are in $\mathrm{cm}^{-1} . \%$ diff $=((\text { calc.-exp. }) / \text { exp. })^{*} 100$. Experimental data are from Refs. 5-8. 
Table S6. Torsional parameters for the Drude polarizable and the additive $\mathrm{C} 27 \mathrm{r}$ alkane force field

\begin{tabular}{lllllll}
\hline Term & \multicolumn{3}{c}{$\mathrm{C} 27 \mathrm{r}$} & \multicolumn{3}{c}{ Drude } \\
\hline & $\mathrm{K} \phi$ & $\mathrm{n}$ & $\delta$ & $\mathrm{K} \phi$ & $\mathrm{n}$ & $\delta$ \\
$\mathrm{CH}_{3}-\mathrm{CH}_{2}-\mathrm{CH}_{2}-\mathrm{CH}_{3}$ & 0.03179 & 6 & 180 & 0.20000 & 5 & 0 \\
& 0.03819 & 2 & 0 & 0.14400 & 4 & 0 \\
& & & & 0.08400 & 3 & 0 \\
& & & & 0.12600 & 2 & 0 \\
& & & & & & \\
$\mathrm{CH}_{3}-\mathrm{CH}_{2}-\mathrm{CH}_{2}-\mathrm{CH}_{2}$ & 0.20391 & 5 & 0 & 0.14200 & 5 & 0 \\
& 0.10824 & 4 & 0 & 0.10500 & 4 & 0 \\
& 0.08133 & 3 & 180 & 0.08200 & 3 & 180 \\
& 0.15051 & 2 & 0 & 0.05300 & 2 & 0 \\
$\mathrm{CH}_{2}-\mathrm{CH}_{2}-\mathrm{CH}_{2}-\mathrm{CH}_{2}$ & 0.11251 & 5 & 0 & 0.13600 & 5 & 0 \\
& 0.09458 & 4 & 0 & 0.07800 & 4 & 0 \\
& 0.14975 & 3 & 180 & 0.13900 & 3 & 180 \\
& 0.06450 & 2 & 0 & 0.08300 & 2 & 0 \\
\hline
\end{tabular}

$\mathrm{K} \phi$ are the force constants in $\mathrm{kcal} / \mathrm{mol}, \mathrm{n}$ is the periodicity or multiplicity and $\delta$ is the phase in degrees. C27r parameters are from Ref. 9. 
Table S7. Relative energies of the barrier heights for the rotation around C-C bonds in alkanes

\begin{tabular}{|c|c|c|c|c|c|}
\hline & $\mathrm{QM}$ & Drude & $\mathrm{dE}$ & $\mathrm{C} 27 \mathrm{r}$ & $\mathrm{dE}$ \\
\hline Ethane & & 2.89 & & 2.90 & \\
\hline Propane & & 3.12 & & 3.12 & \\
\hline \multicolumn{6}{|l|}{ Butane } \\
\hline $\mathrm{A}(\mathrm{T} / \mathrm{G})$ & 3.30 & 3.33 & 0.03 & 3.35 & 0.05 \\
\hline $\mathrm{C}(\mathrm{T} / \mathrm{G})$ & 5.79 & 5.49 & -0.30 & 4.96 & -0.84 \\
\hline \multicolumn{6}{|l|}{ Pentane } \\
\hline TA (TT/TG) & 3.20 & 3.11 & -0.09 & 2.94 & -0.26 \\
\hline TC (TT/TG) & 5.36 & 5.10 & -0.26 & 5.21 & -0.15 \\
\hline $\mathrm{A}^{\prime} \mathrm{G}\left(\mathrm{TG} / \mathrm{G}^{\prime} \mathrm{G}\right)$ & 3.39 & 3.62 & 0.23 & 3.55 & 0.16 \\
\hline $\mathrm{AG}(\mathrm{TG} / \mathrm{GG})$ & 3.51 & 3.82 & 0.31 & 3.52 & 0.01 \\
\hline \multicolumn{6}{|l|}{ Hexane } \\
\hline TAT (TTT/TGT) & 2.91 & 2.97 & 0.06 & 2.91 & 0.00 \\
\hline TCT (TTT/TGT) & 5.26 & 5.05 & -0.21 & 4.90 & -0.36 \\
\hline TGA (TGT/TGG) & 3.48 & 3.70 & 0.22 & 3.45 & -0.03 \\
\hline TGA' (TGT/TGG') & 3.34 & 3.51 & 0.17 & 3.43 & 0.09 \\
\hline GTA (GTT/GTG') & 3.73 & 3.75 & 0.02 & 3.52 & -0.21 \\
\hline GTA' (GTT/GTG) & 3.75 & 3.65 & -0.10 & 3.52 & -0.23 \\
\hline \multicolumn{6}{|l|}{ Heptane } \\
\hline TATT (TTTT/TGTT) & 2.93 & 2.95 & 0.02 & 2.90 & -0.03 \\
\hline TCTT (TTTT/TGTT) & 5.23 & 4.93 & -0.30 & 4.86 & -0.37 \\
\hline TGAT (TGTT/TGGT) & 3.25 & 3.48 & 0.23 & 3.42 & 0.17 \\
\hline TGA'T (TGTT/TGG'T) & 3.10 & 3.23 & 0.13 & 3.25 & 0.16 \\
\hline TGTA (TGTT/TGTG) & 3.71 & 3.69 & -0.02 & 3.47 & -0.24 \\
\hline TGTA' (TGTT/TGTG') & 3.73 & 3.61 & -0.12 & 3.48 & -0.25 \\
\hline
\end{tabular}

Energies are in $\mathrm{kcal} / \mathrm{mol}$ relative to the global minimum for a given molecule. QM data are from Ref. 9. 
Table S8. Comparison of QM and empirical force field minimum interaction energies and geometries between alkane and helium or neon atom.

\begin{tabular}{|c|c|c|c|c|c|c|c|c|c|}
\hline \multirow{2}{*}{\multicolumn{2}{|c|}{ Orientation }} & \multicolumn{3}{|c|}{$\mathrm{R}_{\min }(\AA)$} & \multicolumn{5}{|c|}{$\mathrm{E}_{\min }(\mathrm{mkH})$} \\
\hline & & QM & $\mathrm{C} 27 \mathrm{r}$ & Drude & QM & $\mathrm{C} 27 \mathrm{r}$ & Diff & Drude & Diff \\
\hline \multicolumn{10}{|c|}{ Ethane/helium } \\
\hline 1 & $\mathrm{C}-\mathrm{C}$ & 3.38 & 3.34 & 3.34 & -153.2 & -184.9 & -31.7 & -162.3 & -9.1 \\
\hline 2 & $\mathrm{H}-\mathrm{C}-\mathrm{H}$ & 3.69 & 3.38 & 3.38 & -111.9 & -197.4 & -85.5 & -175.2 & -63.3 \\
\hline 3 & $\mathrm{C}-\mathrm{H}$ & 2.88 & 2.66 & 2.66 & -112.2 & -155.6 & -43.4 & -133.5 & -21.3 \\
\hline \multirow[t]{4}{*}{4} & C-C norm & 3.61 & 3.44 & 3.44 & -165.9 & -245.2 & -79.3 & -222.9 & -57.0 \\
\hline & RMSD & & 0.098 & 0.098 & & 22.9 & & 23.0 & \\
\hline & RMSR & & 0.028 & 0.028 & & 0.201 & & 0.188 & \\
\hline & AAE & & 0.185 & 0.185 & & 60.0 & & 37.7 & \\
\hline \multicolumn{10}{|c|}{ Propane/helium } \\
\hline 1 & $\mathrm{C} 2-\mathrm{C} 1$ & 4.18 & 3.89 & 3.91 & -136.4 & -189.8 & -53.4 & -182.2 & -45.7 \\
\hline 2 & $\mathrm{H}-\mathrm{C} 2-\mathrm{H}$ & 3.66 & 3.33 & 3.33 & -129.1 & -217.8 & -88.7 & -219.2 & -90.1 \\
\hline 3 & $\mathrm{H}-\mathrm{C} 2$ & 2.85 & 2.64 & 2.65 & -131.4 & -165.5 & -34.0 & -162.0 & -30.6 \\
\hline 4 & $\mathrm{C} 2-\mathrm{H}$ & 4.21 & 3.85 & 3.84 & -175.3 & -278.0 & -102.8 & -272.9 & -97.6 \\
\hline 5 & $\mathrm{C}-\mathrm{C}-\mathrm{C}$ & 4.32 & 3.99 & 3.98 & -193.1 & -320.1 & -127.0 & -314.3 & -121.2 \\
\hline 6 & $\mathrm{H}-\mathrm{C} 1$ & 2.88 & 2.66 & 2.65 & -128.0 & -143.2 & -15.2 & -136.4 & -8.5 \\
\hline \multirow[t]{4}{*}{7} & $\mathrm{C} 1-\mathrm{C} 2$ & 3.38 & 3.33 & 3.33 & -156.5 & -176.7 & -20.2 & -168.7 & -12.1 \\
\hline & RMSD & & 0.099 & 0.101 & & 40.3 & & 41.5 & \\
\hline & RMSR & & 0.023 & 0.024 & & 0.225 & & 0.241 & \\
\hline & AAE & & 0.256 & 0.256 & & 63.0 & & $\mathbf{5 8 . 0}$ & \\
\hline \multicolumn{10}{|c|}{ Isobutane/helium } \\
\hline 1 & HT-CT & 2.84 & 2.62 & 2.64 & -149.1 & -132.1 & 17.0 & -186.6 & -37.5 \\
\hline 2 & CT-HT & 4.23 & 3.84 & 3.81 & -242.8 & -310.0 & -67.1 & -357.1 & -114.3 \\
\hline 3 & C1-CT-HT & 4.17 & 3.99 & 3.98 & -170.2 & -175.1 & -4.9 & -223.6 & -53.3 \\
\hline 4 & $\mathrm{C} 1-\mathrm{CT}-\mathrm{C} 2$ & 4.32 & 4.01 & 4.00 & -204.8 & -275.2 & -70.4 & -317.8 & -113.0 \\
\hline 5 & $\mathrm{H} 11-\mathrm{C} 1$ & 2.86 & 2.64 & 2.63 & -134.6 & -127.2 & 7.4 & -168.4 & -33.8 \\
\hline \multirow[t]{4}{*}{6} & $\mathrm{C} 1-\mathrm{CT}$ & 3.38 & 3.34 & 3.33 & -167.5 & -133.9 & 33.6 & -174.1 & -6.6 \\
\hline & RMSD & & 0.109 & 0.115 & & 40.4 & & 40.5 & \\
\hline & RMSR & & 0.027 & 0.027 & & 0.199 & & 0.165 & \\
\hline & AAE & & 0.227 & 0.235 & & 33.4 & & 59.8 & \\
\hline
\end{tabular}

QM energies and geometries for alkane-rare gas complexes are calculated at MP3/6-311++G(3d,3p) level of theory using MP2/6-31G(d) optimized geometries of alkane. RMSD - root mean square difference. RMSR - root mean square ratio. The orientation indicates the direction of the $\mathrm{He} / \mathrm{Ne}$ atom approach: X1-X2 means along X1-X2 bond from the side of X1 atom; X1-X2-X3 means along the $\mathrm{X} 1-\mathrm{X} 2-\mathrm{X} 3$ angle bisector; $\mathrm{X} 1-\mathrm{X} 2$ norm means perpendicular to the $\mathrm{X} 1-\mathrm{X} 2$ bond. 
Table S8 (continued).

\begin{tabular}{|c|c|c|c|c|c|c|c|c|c|}
\hline \multirow{2}{*}{\multicolumn{2}{|c|}{ Orientation }} & \multicolumn{3}{|c|}{$\mathrm{R}_{\min }(\AA)$} & \multicolumn{5}{|c|}{$\mathrm{E}_{\min }(\mathrm{mkH})$} \\
\hline & & QM & $\mathrm{C} 27 \mathrm{r}$ & Drude & QM & $\mathrm{C} 27 \mathrm{r}$ & Diff & Drude & Diff \\
\hline \multicolumn{10}{|c|}{ Ethane/neon } \\
\hline 1 & $\mathrm{C}-\mathrm{C}$ & 3.32 & 3.38 & 3.38 & -862.7 & -352.8 & 509.9 & -330.0 & 532.7 \\
\hline 2 & $\mathrm{H}-\mathrm{C}-\mathrm{H}$ & 3.50 & 3.43 & 3.43 & -669.8 & -377.1 & 292.7 & -355.1 & 314.7 \\
\hline 3 & $\mathrm{C}-\mathrm{H}$ & 2.76 & 2.70 & 2.70 & -720.9 & -294.0 & 426.9 & -272.1 & 448.8 \\
\hline \multirow[t]{4}{*}{4} & C-C norm & 3.49 & 3.49 & 3.49 & -900.3 & -473.0 & 427.3 & -450.8 & 449.6 \\
\hline & RMSD & & 0.052 & 0.052 & & 77.9 & & 78.1 & \\
\hline & RMSR & & 0.016 & 0.016 & & 0.069 & & 0.069 & \\
\hline & $\mathbf{A A E}$ & & 0.048 & 0.048 & & 414.2 & & 436.4 & \\
\hline \multicolumn{10}{|c|}{ Propane/neon } \\
\hline 1 & $\mathrm{C} 2-\mathrm{C} 1$ & 4.01 & 3.94 & 3.97 & -822.1 & -376.2 & 445.9 & -369.3 & 452.8 \\
\hline 2 & $\mathrm{H}-\mathrm{C} 2-\mathrm{H}$ & 3.47 & 3.37 & 3.37 & -820.3 & -433.9 & 386.4 & -445.3 & 375.0 \\
\hline 3 & $\mathrm{H}-\mathrm{C} 2$ & 2.73 & 2.68 & 2.69 & -827.9 & -329.9 & 498.0 & -331.3 & 496.6 \\
\hline 4 & $\mathrm{C} 2-\mathrm{H}$ & 4.04 & 3.90 & 3.89 & -1001.7 & -552.9 & 448.8 & -550.8 & 450.8 \\
\hline 5 & $\mathrm{C}-\mathrm{C}-\mathrm{C}$ & 4.07 & 4.04 & 4.03 & -1162.0 & -638.9 & 523.1 & -635.2 & 526.8 \\
\hline 6 & $\mathrm{H}-\mathrm{C} 1$ & 2.74 & 2.70 & 2.70 & -803.7 & -283.7 & 519.9 & -278.5 & 525.2 \\
\hline \multirow[t]{4}{*}{7} & $\mathrm{C} 1-\mathrm{C} 2$ & 3.30 & 3.38 & 3.38 & -937.9 & -351.2 & 586.7 & -343.3 & 594.6 \\
\hline & RMSD & & 0.064 & 0.065 & & 60.6 & & 65.1 & \\
\hline & RMSR & & 0.018 & 0.018 & & 0.079 & & 0.083 & \\
\hline & $\mathbf{A A E}$ & & 0.073 & 0.070 & & 487.0 & & 488.8 & \\
\hline \multicolumn{10}{|c|}{ Isobutane/neon } \\
\hline 1 & HT-CT & 2.71 & 2.66 & 2.69 & -949.3 & -314.9 & 634.4 & -382.6 & 566.7 \\
\hline 2 & CT-HT & 4.05 & 3.89 & 3.87 & -1241.4 & -667.7 & 573.7 & -719.6 & 521.9 \\
\hline 3 & C1-CT-HT & 4.03 & 4.04 & 4.03 & -1045.9 & -398.4 & 647.5 & -454.2 & 591.7 \\
\hline 4 & $\mathrm{C} 1-\mathrm{CT}-\mathrm{C} 2$ & 4.09 & 4.07 & 4.05 & -1230.9 & -599.5 & 631.4 & -643.0 & 587.9 \\
\hline 5 & $\mathrm{H} 11-\mathrm{C} 1$ & 2.72 & 2.68 & 2.67 & -852.6 & -304.4 & 548.2 & -345.4 & 507.2 \\
\hline \multirow[t]{4}{*}{6} & $\mathrm{C} 1-\mathrm{CT}$ & 3.29 & 3.37 & 3.37 & -1010.5 & -316.2 & 694.3 & -354.8 & 655.6 \\
\hline & RMSD & & 0.072 & 0.077 & & 48.1 & & 49.0 & \\
\hline & RMSR & & 0.020 & 0.021 & & 0.083 & & 0.078 & \\
\hline & AAE & & 0.060 & 0.062 & & 621.6 & & $\mathbf{5 7 1 . 8}$ & \\
\hline
\end{tabular}

QM energies and geometries for alkane-rare gas complexes are calculated at MP3/6-311++G(3d,3p) level of theory using MP2/6-31G(d) optimized geometries of alkane. RMSD - root mean square difference. RMSR - root mean square ratio. AAE - average absolute error. . The orientation indicates the direction of the $\mathrm{He} / \mathrm{Ne}$ atom approach: X1-X2 means along X1-X2 bond from the side of X1 atom; X1-X2-X3 means along the X1-X2-X3 angle bisector; X1-X2 norm means perpendicular to the X1-X2 bond. 
Table S9. Parameters of the nonpolarizable TIP3P $\mathrm{P}^{10}$ and Drude polarizable SWM4-NDP ${ }^{11}$ water models used in the determination of the solvation free energies of alkanes.

\begin{tabular}{lrr}
\hline & TIP3P & SWM4-N \\
\hline \multicolumn{2}{l}{ Internal parameters } & \\
$l_{\mathrm{OH}}(\AA)$ & 0.9572 & 0.9572 \\
$\theta_{\mathrm{HOH}}\left({ }^{\circ}\right)$ & 104.52 & 104.52 \\
$l_{\mathrm{OM}}(\AA)$ & & 0.24034 \\
Electrostatic parameters & \\
$q_{\mathrm{O}}(\mathrm{e})$ & -0.83400 & 1.71636 \\
$q_{\mathrm{M}}(\mathrm{e})$ & & -1.11466 \\
$q_{\mathrm{H}}(\mathrm{e})$ & 0.41700 & 0.55733 \\
$q_{\mathrm{D}}(\mathrm{e})$ & & -1.71636 \\
$k_{\mathrm{D}}\left(\mathrm{kcal} / \mathrm{mol} / \AA^{2}\right)$ & & 1000 \\
Lennard-Jones parameters & \\
$\varepsilon_{\mathrm{O}}(\mathrm{kcal} / \mathrm{mol})$ & 0.15210 & 0.21094 \\
$\sigma_{\mathrm{O}}(\AA)$ & 3.1506 & 3.18395 \\
$\varepsilon_{\mathrm{H}}(\mathrm{kcal} / \mathrm{mol})$ & 0.04600 & 0.00000 \\
$\sigma_{\mathrm{H}}(\AA)$ & 0.4490 & 0.0000 \\
\hline
\end{tabular}




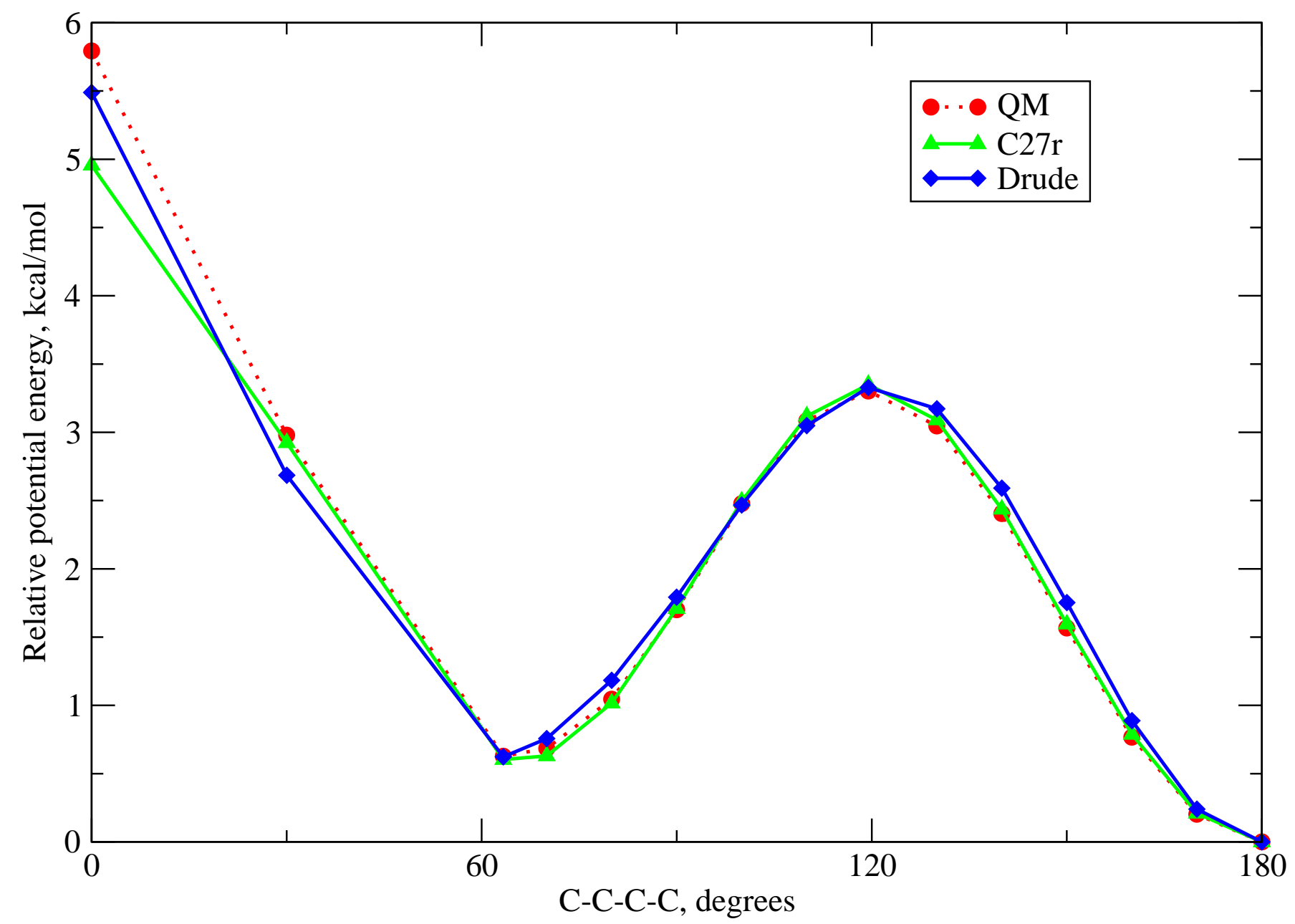

Figure S1. Empirical and QM adiabatic energy surfaces for rotation around C-C-C-C dihedral angle in butane. Potential energies are given with respect to the global minimum (trans conformer). QM data are from Klauda et al. ${ }^{9}$ 


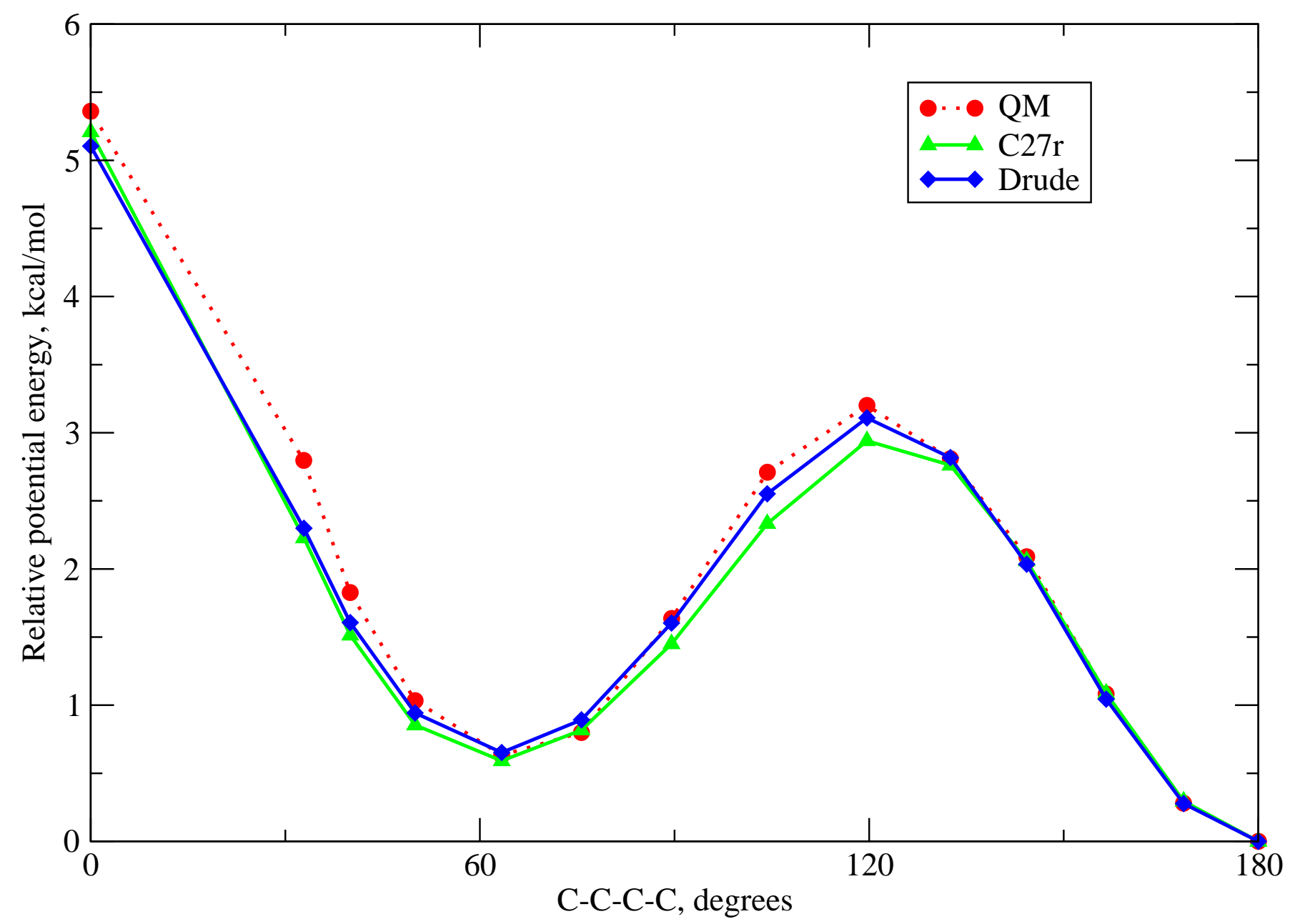

Figure S2. Empirical and QM adiabatic energy surfaces for rotation around the $\mathrm{C} 1-\mathrm{C} 2-\mathrm{C} 3-\mathrm{C} 4$ dihedral angle in pentane. The dihedral angle $\mathrm{C} 2-\mathrm{C} 3-\mathrm{C} 4-\mathrm{C} 5$ is in the trans conformation. Potential energies are given with respect to the global minimum ( $t t$ conformer). QM data are from Klauda et al. ${ }^{9}$ 


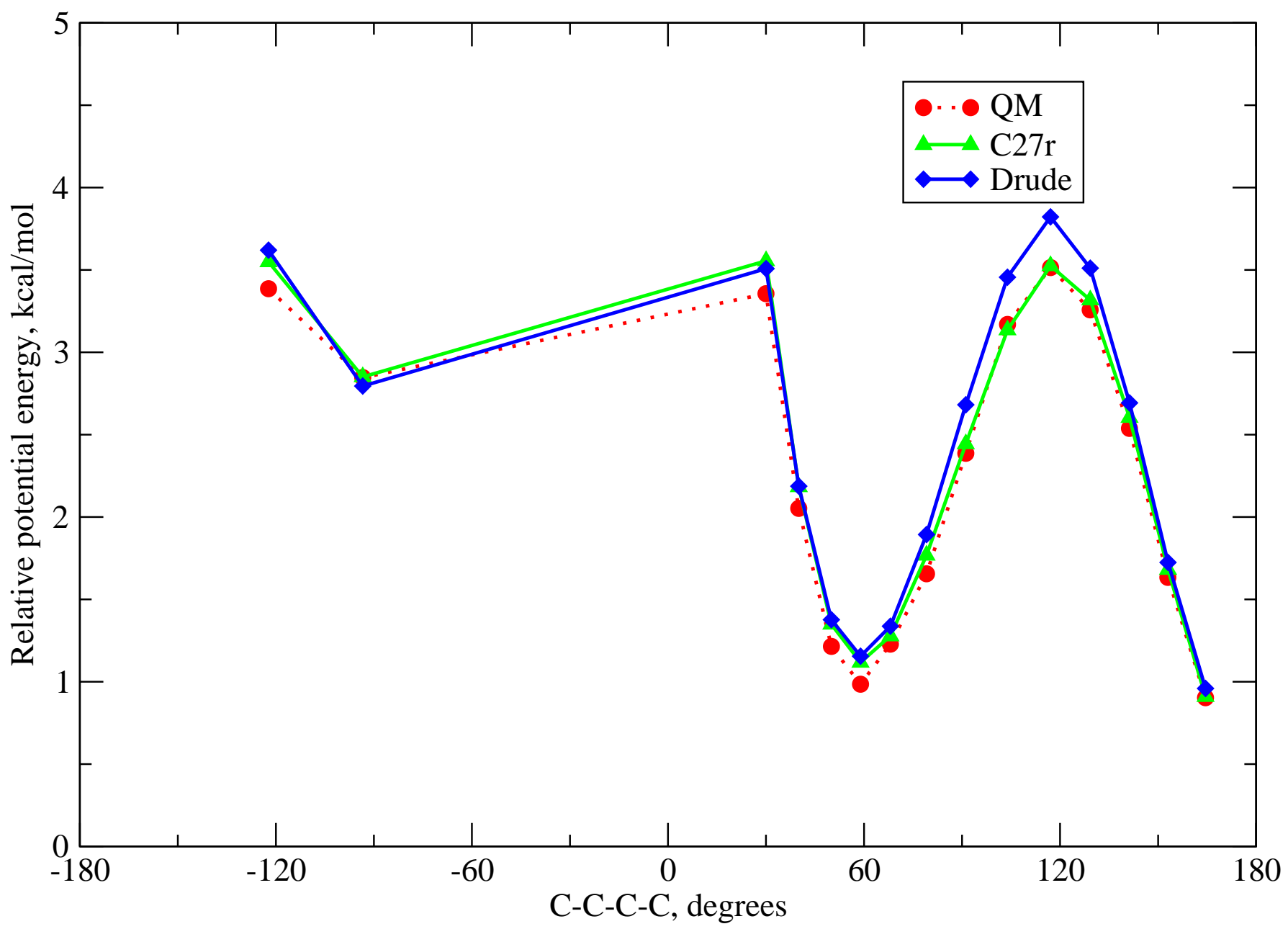

Figure S3. Empirical and QM adiabatic energy surfaces for rotation around the $\mathrm{C} 2-\mathrm{C} 3-\mathrm{C} 4-\mathrm{C} 5$ dihedral angle in pentane. The dihedral angle $\mathrm{C} 1-\mathrm{C} 2-\mathrm{C} 3-\mathrm{C} 4$ is in the gauche conformation. Potential energies are given with respect to the global minimum ( $t t$ conformer). QM data are from Klauda et al. ${ }^{9}$ 


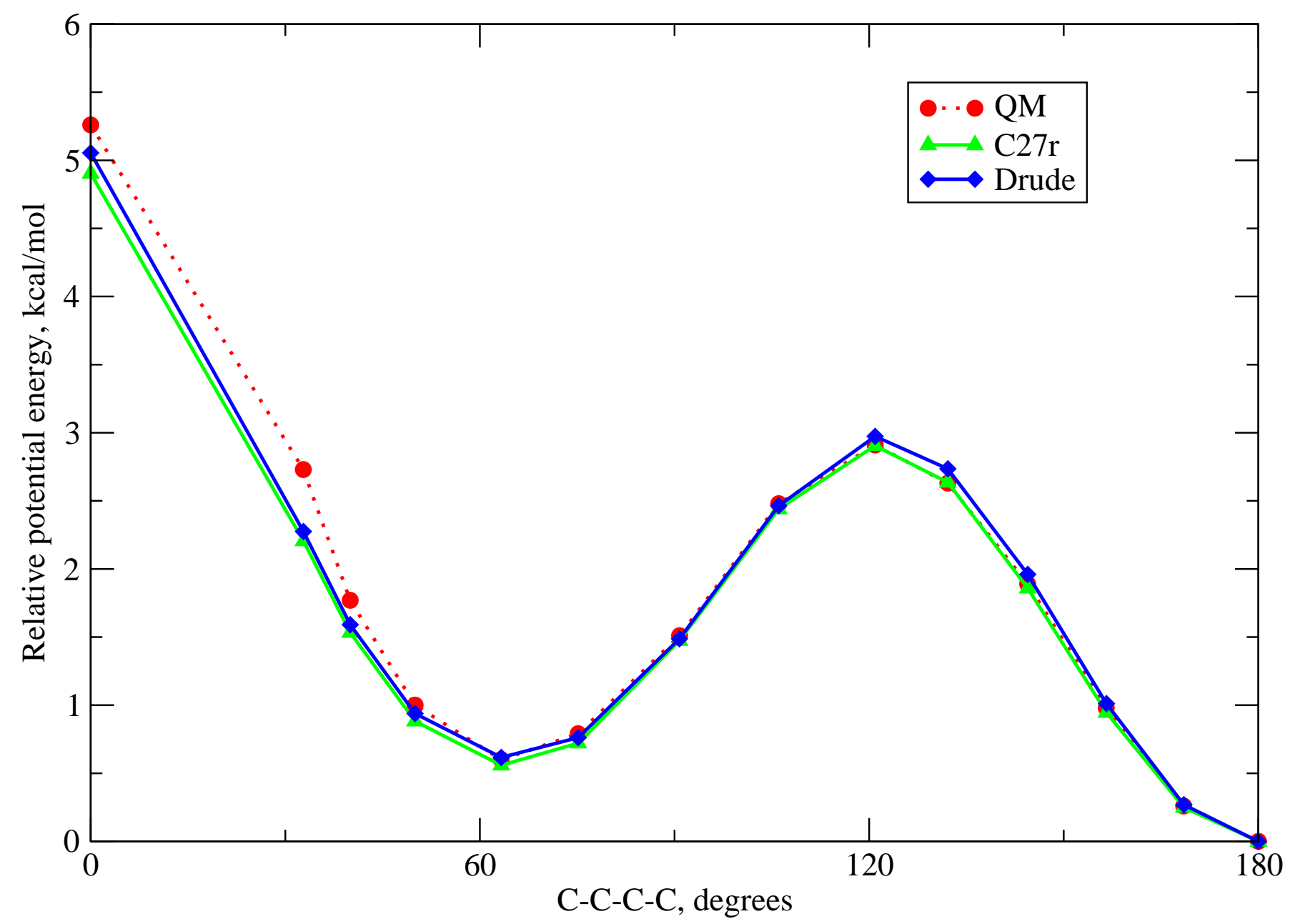

Figure S4. Empirical and QM adiabatic energy surfaces for rotation around the $\mathrm{C} 2-\mathrm{C} 3-\mathrm{C} 4-\mathrm{C} 5$ dihedral angle in hexane. The dihedral angles $\mathrm{C} 1-\mathrm{C} 2-\mathrm{C} 3-\mathrm{C} 4$ and $\mathrm{C} 3-\mathrm{C} 4-\mathrm{C} 5-\mathrm{C} 6$ are in the trans conformation. Potential energies are given with respect to the global minimum (ttt conformer). QM data are from Klauda et al. . $^{9}$ 


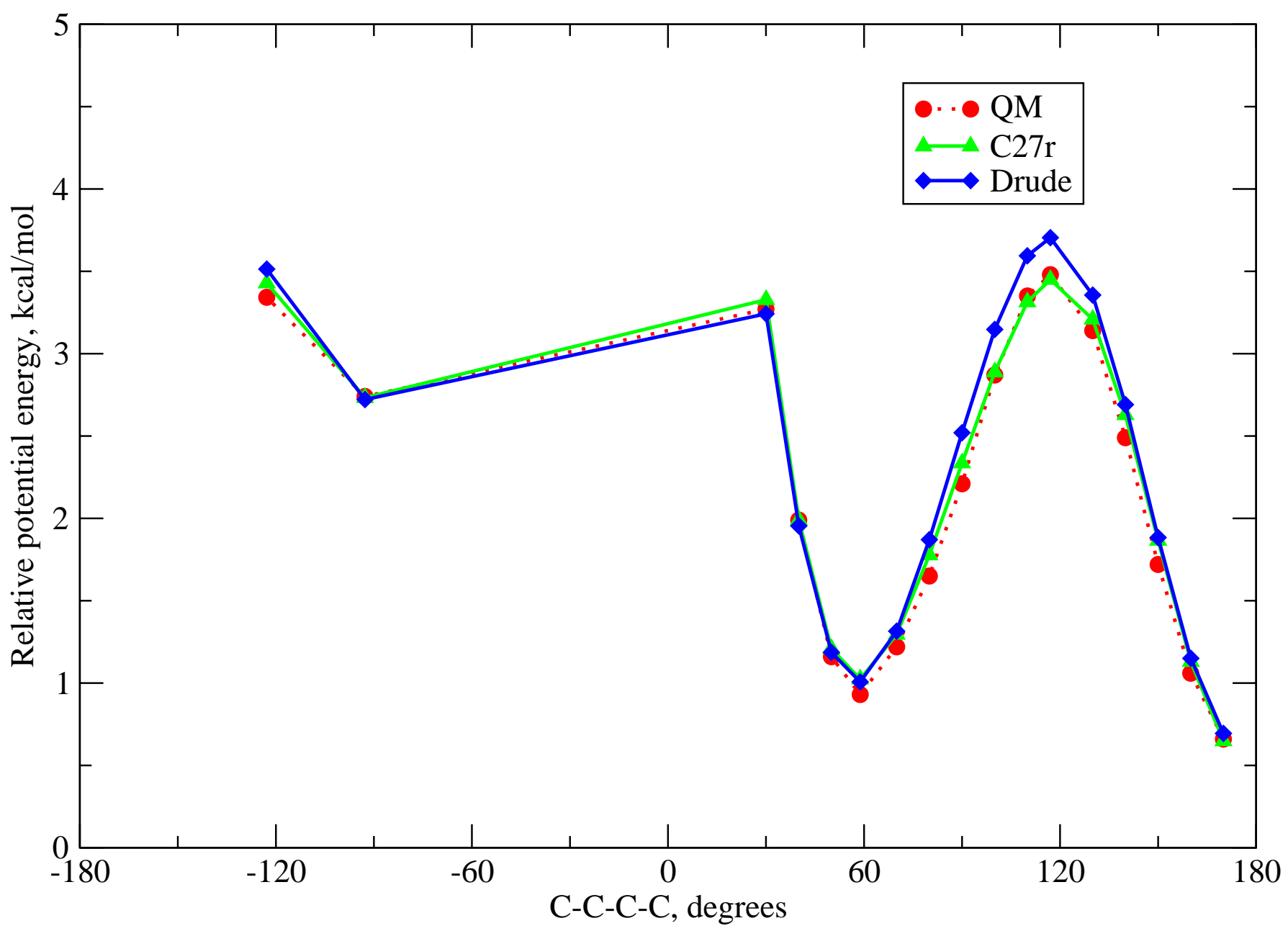

Figure S5. Empirical and QM adiabatic energy surfaces for rotation around C3-C4-C5-C6 dihedral angle in hexane. The dihedral angle $\mathrm{C} 1-\mathrm{C} 2-\mathrm{C} 3-\mathrm{C} 4$ is in the trans and $\mathrm{C} 2-\mathrm{C} 3-\mathrm{C} 4-\mathrm{C} 5$ is in the gauche conformation. Potential energies are given with respect to the global minimum (ttt conformer). QM data are from Klauda et al. ${ }^{9}$ 


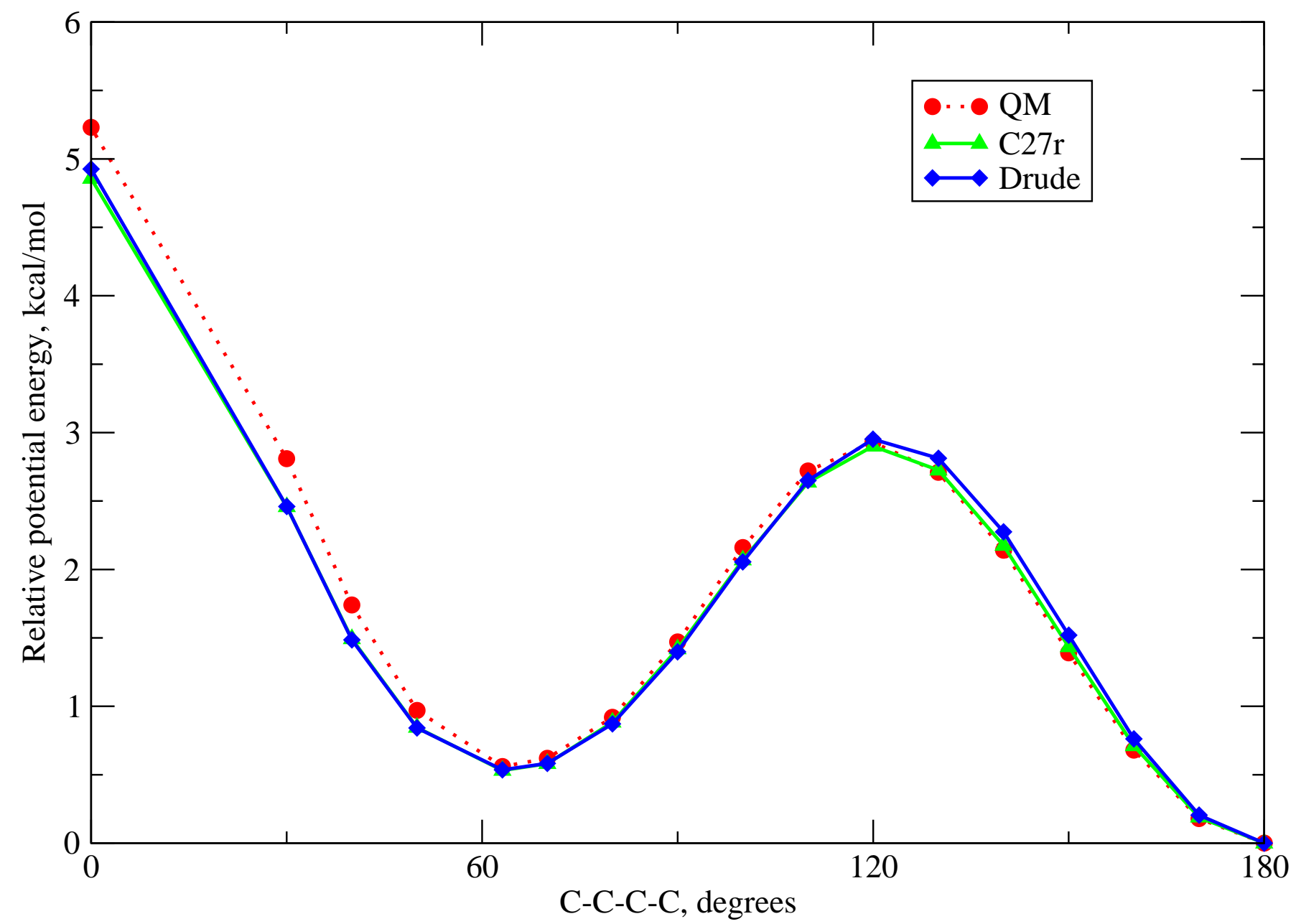

Figure S6. Empirical and QM adiabatic energy surfaces for rotation around $\mathrm{C} 2-\mathrm{C} 3-\mathrm{C} 4-\mathrm{C} 5$ dihedral angle in heptane. The dihedral angles $\mathrm{C} 1-\mathrm{C} 2-\mathrm{C} 3-\mathrm{C} 4, \mathrm{C} 3-\mathrm{C} 4-\mathrm{C} 5-\mathrm{C} 6$, and $\mathrm{C} 4-\mathrm{C} 5-\mathrm{C} 6-\mathrm{C} 7$ are in the trans conformation. Potential energies are given with respect to the global minimum (tttt conformer). QM data are from Klauda et al. ${ }^{9}$ 


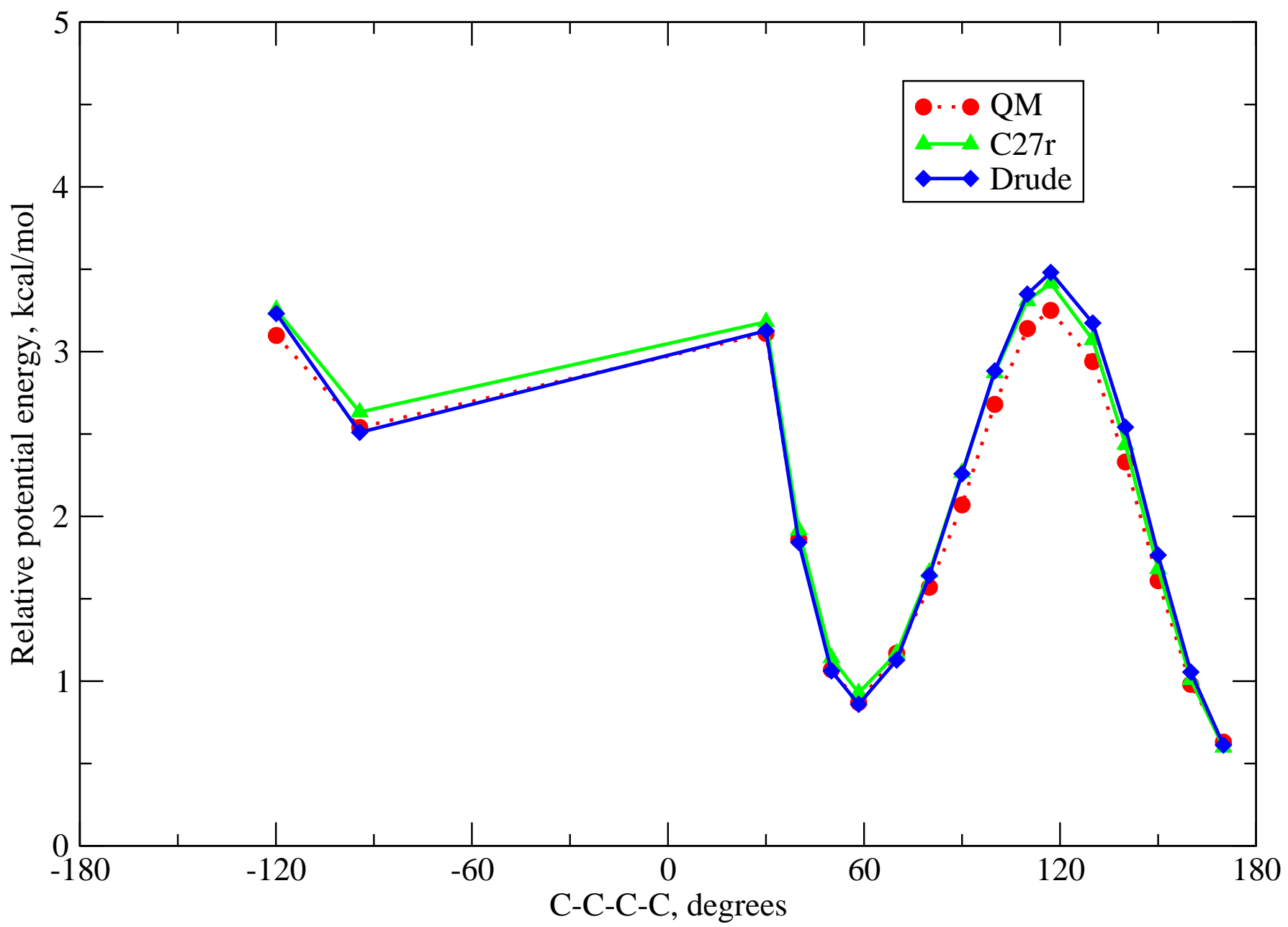

Figure S7. Empirical and QM adiabatic energy surfaces for rotation around $\mathrm{C} 3-\mathrm{C} 4-\mathrm{C} 5-\mathrm{C} 6$ dihedral angle in heptane. The dihedral angles $\mathrm{C} 1-\mathrm{C} 2-\mathrm{C} 3-\mathrm{C} 4$ and $\mathrm{C} 4-\mathrm{C} 5-\mathrm{C} 6-\mathrm{C} 7$ are in the trans, whereas $\mathrm{C} 2-\mathrm{C} 3-\mathrm{C} 4-\mathrm{C} 5$ is in the gauche disposition. Potential energies are given with respect to that of the global minimum (tttt conformer). The QM calculations are from Klauda et al. ${ }^{9}$ 


\section{References}

(1) Hoyland, J. R. J. Chem. Phys. 1969, 50, 2775.

(2) Wiberg, K. B.; Boyd, R. H. J. Am. Chem. Soc. 1972, 94, 8426.

(3) Heenan, R. K.; Bartell, L. S. J. Chem. Phys. 1983, 78, 1270.

(4) Bradford, W. F.; Fitzwater, S.; Bartell, L. S. J. Mol. Struct. 1977, 38, 185.

(5) NIST Computational Chemistry Comparison and Benchmark Database, NIST Standard Reference Database Number 101; 11 ed.; Johnson, R. D., III, Ed.; National Institute of Standards and Technology: Gaithersburg MD, 20899 (http://srdata.nist.gov/cccbdb), 2005.

(6) Lifson, S.; Warshel, A. J. Chem. Phys. 1968, 49, 5116.

(7) Schachtschneider, J. H.; Snyder, R. G. Spectrochimica Acta 1963, 19, 117.

(8) Snyder, R. G.; Schachtschneider, J. H. Spectrochimica Acta 1965, 21, 169.

(9) Klauda, J. B.; Brooks, B. R.; MacKerell, A. D.; Venable, R. M.; Pastor, R. W. J. Phys. Chem. B 2005, 109, 5300 .

(10) Jorgensen, W. L.; Chandrasekhar, J.; Madura, J. D.; Impey, R. W.; Klein, M. L. J. Chem. Phys. 1983, 79, 926.

(11) Lamoureux, G.; Harder, E.; Vorobyov, I.; Deng, Y.; Roux, B.; MacKerell Jr., A. D. (in preparation) 2005. 